Dear Author,

Please, note that changes made to the HTML content will be added to the article before publication, but are not reflected in this PDF.

Note also that this file should not be used for submitting corrections. 


\title{
Acute effects of jaw clenching using a customized mouthguard on anaerobic ability and ventilatory flows
}

\author{
Jose Morales*, Bernat Buscà, Mònica Solana-Tramunt, Adrià Miró \\ Faculty of Psychology, Education Sciences and Sport Blanquerna - Ramon Llull University, c/Císter, 34, 08022 Barcelona, Spain
}

\section{A R T I C L E I N F O}

\section{Article history:}

Received 14 July 2015

Revised 21 September 2015

Accepted 21 September 2015

Available online $\mathrm{xxxx}$

\section{Keywords:}

Mouthguard

Ventilation

Anaerobic power

Ergogenic effects

\begin{abstract}
A B S T R A C T
The latest findings on the ergogenic effects of a dentistry-design, bite-aligning mouthpiece require additional research to assess its impact on anaerobic ability and ventilatory parameters. This paper was aimed at determining the ergogenic acute effects of wearing a custom-made mouthpiece on oral airflow dynamics, 30-s Wingate Anaerobic Test performance parameters.

Twenty-eight healthy and physically-active male subjects (age: $24.50 \pm 3.32$, height: $181.34 \pm 7.4$, weight: $78.14 \pm 8.21$ ), were voluntarily studied. The subjects were first briefed on the test protocols, and then performed the $30 \mathrm{~s}$ Wingate test and Spirometer test. The experimental trials were performed in a random counterbalanced order. We evaluate maximum expiratory volume $\left(V_{\mathrm{Emax}} \mathrm{L} \mathrm{min}^{-1}\right)$, mean power $\left(\mathrm{W} \mathrm{kg}^{-1}\right)$, peak power $\left(\mathrm{W} \mathrm{kg}^{-1}\right)$, time to peak (s), rate to fatigue $\left(\mathrm{W} \mathrm{s}^{-1}\right)$ and lactate production ( $\left.\mathrm{mMol} \mathrm{L}^{-1}\right)$, rate of perceived exertion (RPE).

There were significant differences between mouthguard and no-mouthguard conditions in mean power $\left(\mathrm{W} \mathrm{kg}^{-1}\right)$, peak power $\left(\mathrm{W} \mathrm{kg}^{-1}\right)$, time to peak (s), and rate to fatigue $\left(\mathrm{W} \mathrm{s}^{-1}\right)$ for the 30-s Wingate Anaerobic Test. Significantly lower lactate production ( $\mathrm{mMol} \mathrm{L}^{-1}$ ) was observed, in mouthguard condition but no significant differences were found in RPE. In airflow dynamics, the $V_{\mathrm{Emax}} \mathrm{L} \mathrm{min}^{-1}$ was significantly higher when comparing the mouthguard and the no mouthguard conditions in both forced and unforced conditions.

In conclusion, wearing a customized mouthguard improves anaerobic ability and increases forced expiratory volume. This study will help practitioners improve athlete's performance in anaerobic activities where high intensity action might provoke jawclenching, contributing in reductions of lactate and fatigue, and improving ventilatory parameters.
\end{abstract}

(c) 2015 Published by Elsevier B.V.

\section{Introduction}

In some sports, athletes use different types of mouthguards to prevent dental injuries and to protect the maxillofacial structure from possible shocks and collisions. Mouthguards allow athletes to feel protected, but the devices are not always comfortable. There are three main types of mouthguards: standard, self-adapted, and customized. Standard mouth guards are a fitted piece that requires no adjustments. Due to its relatively low cost, it is the most commonly used mouthguard,

\footnotetext{
* Corresponding author.

E-mail addresses: josema@blanquerna.url.edu (J. Morales), bernatbs@blanquerna.url.edu (B. Buscà), monicast2@blanquerna.url.edu (M. SolanaTramunt), adriama@blanquerna.url.edu (A. Miró).
} 
but is often uncomfortable. Self-adapted mouthguards consist of a "thermoplastic" liner that can be manipulated with heat during an at-home fitting process to improve the fit to the maxillary teeth. This type is also inexpensive and widely available. Finally, customized mouthguards require dental impressions or use of a scanning process of the dental structure of an individual's teeth. This is the most expensive of the three types of mouthguards and often requires the expertise of a dentist (Chalmers, 1998; Geary, Clifford, \& Kinirons, 2009; Patrick, van Noort, \& Found, 2005). Beyond the preventive role of wearing mouthguards, recent research has investigated its physiological effects and the possible benefits in different parameters.

Although it has been demonstrated that wearing a mouthguard reduces orofacial injury, many athletes do not wear them during training sessions or in competition for different reasons, including speech and breathing difficulties or discomfort (Collares, Correa, Da Silva, Hallal, \& Demarco, 2013; Duarte-Pereira et al., 2008; Gebauer, Williamson, Wallman, \& Dawson, 2011). In recent years, several researchers have studied the effects of wearing mouthguards on physiological and breathing parameters in an attempt to demonstrate that they are unlikely to interfere with an athlete's performance. Francis and Brasher (1991) studied the effect of three different types of mouthguards on forced expiratory volume, maximum flow rate in a single forced expiration, subject's $\mathrm{VO}_{2} \max$, and one-minute ventilation of expired gases collected during a cycling exercise. The authors found that wearing mouthguards produced an effective respiratory pattern during brief periods of heavy exercise, which might improve tissue oxygenation and lower metabolic cost (Francis \& Brasher, 1991). Amis et al. (2000) studied the influence of intra-oral maxillary sports mouthguards on the airflow dynamics of oral breathing and found that mouthguards cause obstruction to oral breathing, mainly at rest and when the degree of mouth opening is restricted; however, these are unlikely to cause interference at high ventilatory rates (Amis, Di Somma, Bacha, \& Wheatley, 2000). Other studies have found no difference in oxygen consumption and ventilation in trained subjects (Bourdin et al., 2006; Collares et al., 2013; Gebauer et al., 2011; Rapisura, Coburn, Brown, \& Kersey, 2010).

For some sports, ventilatory mechanisms are closely connected to the anaerobic recovery phases. Thus, wearing a mouthguard wouldn't hamper oxygen intake during these phases. Furthermore, mechanisms like concurrent activation potentiation (CAP) that are derived from a clenched jaw and which constitute a remote voluntary contraction (RVC) have been suggested as the possible causes of an ergogenic effect of wearing mouthguards (Ebben, 2006; Ebben, Flanagan, \& Jensen, 2008). The neuromuscular effects of jaw-repositioning and contraction of the mandible muscles may translate to improved neuromuscular responses in active exercise movers. As a consequence, the activation of different limb muscles contribute to strengthen movements like rowing, pedaling, running, or jumping (Milani, De Periere, Lapeyre, \& Pourreyron, 2000). When one part of the motor cortex is active, connections to other areas of the motor cortex are also affected (Ebben, 2006). Thus, CAP has been suggested as a performance enhancer with regards to several acute power and strength tasks in sports thanks to the RVC of jaw muscles (Ebben, 2006; Ebben, Leigh, \& Geiser, 2008; Forgione, Mehta, \& Westcott, 1991). For instance, analyzing the muscle activation via electromyography in a group of healthy and active men and women, the muscles involved in a RVC are more active; this increase in activity results in a greater activity in the prime movers in isokinetic knee extensionflexion (Ebben, Kaufmann, Fauth, \& Petushek, 2010). Furthermore, it has been suggested that biting a custom made mouthguard could enhance athletic performance in quick powerful and anaerobic actions and increase ventilatory pathways. Garner and McDivitt (2009) used computer tomography scans and found that the use of a jaw-repositioning mouthpiece increased oropharynx width and diameter, and concluded that the increased airway opening promoted improved gas exchange in endurance athletes. Arent et al. (2010) compared the effects of a non-dentistry design mouthguard and a traditional custom-fitted mouthguard and found significant differences in countermovement vertical jump (CMVJ) height and peak power in a $30 \mathrm{~s}$ Wingate Anaerobic Test (WAnT). They used transcutaneous electrical nerve stimulation (TENS) to ensure relaxation of the lower jaw before the fitting process of the mouthguards. Cetin et al. (2009) also found significant increases in relative peak and average power in WAnT for elite taekwondo athletes who wore a mouthguard, compared to those who wore no mouthguard. Dunn-Lewis et al. (2012) compared the effects of three conditions (customized, self-fitted, and no mouthguard) on different power performance tests. They reported significantly higher power production in plyo press power quotient (i.e., a more specific anaerobic performance test than WAnT) and higher rates of power development in CMJV for men who used a customized mouthguard. In contrast, Bourdin et al. (2006) found no significant differences in force and power parameters in a cycle ergometer, for trained men who used customized and self-adapted mouthguards. Other studies found no significant improvements of performance in anaerobic ability tests (Bailey et al., 2014; DuartePereira et al., 2008; Duddy et al., 2012).

The latest findings on the ergogenic effects of a dentistry-design, bite-aligning mouthpiece require additional research to assess its impact on anaerobic ability and ventilatory parameters. Therefore, this study sought to investigate the acute effects of a customized, bite-aligning mouthpiece on the different parameters assessed during a WnAT, as compared to clenching the jaw without a mouthpiece, in a physically-active, male population. The study also investigated the influence of wearing a mouthpiece on non-forced and forced airflow dynamics, as compared to open mouth and a jaw clenching without mouthpiece. Mouthpieces were made using a new scanning method that simplified the fitting process and lowered its cost.

\section{Methods}

Twenty-eight healthy and physically-active male subjects (age: $24.50 \pm 3.32$, height: $181.34 \pm 7.4$, weight: $78.14 \pm 8.21$, body mass index: $24.81 \pm 1.47$ ), were voluntarily recruited for this study. All participants were physically active in different disciplines (from 3 to 4 training sessions per week of soccer, handball, basketball or field hockey), competed in regional 
championships and were free of temporomandibular joint disorders. The subjects were required to stop training sessions at least $24 \mathrm{~h}$ before the testing sessions. They were also asked not to intake any stimulating substance (caffeine, theine...) and alcohol drinks one day before. The study was performed with informed consent and following all the guidelines for experimental investigation with humans. The ethics committee of the Ramon Llull University of Barcelona approved the development of this study which was conducted according to the latest version of declaration of Helsinki.

\subsection{Experimental overview}

Each subject participated in three sessions. The first session was used to assess anthropometric measurements and to scan the subject's mouth structure. In this session, an expert dentist evaluated the possible temporomandibular joint disorders and the quality of occlusion. Subjects with a consistent malocclusion were excluded from the study. In the second session, subjects were familiarized with the test protocols via a learning session that included demonstration of WnAT and the airflow dynamics measurements. Subjects also performed the airflow tests and the first WAnT trial. In the third session, subjects performed the second WnAT trial. Conditions were randomly distributed in all tests. WnAT trials were separated by three days and subjects were not allowed to perform intensive training workloads.

The intraoral device CleverBite ${ }^{\circledR}$ (Cleverbite SL, Terrassa, Spain) is a mouthguard Class III (American Society for Testing \& Materials, 2000), and is manufactured by taking a digital recording of a scan of both the maxillary and mandibular dental arches (Fig. 1). This record is complemented by a digital recording of the interocclusal relation associated with the resting position of the mandible. The device fabrication is performed on a digital model made of Pearlstone Visijet Plastic Material (Urethane Acrylate and Phenylbis (2, 4, 6-trimethylbenzoyl) phosphine oxide) obtained by scanning the dentoalveolar maxillary and mandibular environment using the 3Shape Trios System (3Shape Inc. Copenhagen, Denmark). Prior the scanning, subjects performed different maneuvers to neutralize any postural neuromuscular disorders that might influence the centric mandible position with respect to cranio and the cervical muscle activation. Subjects wore a $2-\mathrm{cm}$ long and $0.5-\mathrm{cm}$ thick cylindrical piece of cotton in both sides of the mouth (i.e., at the level from first molar to the distal slope of the canine cusped) and were asked to walk barefoot for $2 \mathrm{~min}$. After walking, subjects performed neck rotations, flexo-extensions, and lateral flexions for 1 min each.

\subsubsection{Wingate Anaerobic Test}

Anaerobic power and capacity were assessed using the 30-s WAnT with and without mouthguard (MOUTHG and NOMOUTHG, respectively). Subjects completed a 30-s maximal effort on an electronically braked cycle ergometer Lode Excalibur Sport V2.0 (Lode Medical Technology, Groningen, The Netherlands) at a resistance equivalent to $7.5 \%$ of their body mass. The ergometer was interfaced with a computer loaded with software (Wingate Software Version 1.11, Lode BV) that applied the appropriate load for each subject. As a warm-up procedure, subjects were instructed to begin pedaling for 5 min at $100 \mathrm{~W}$ and approximately $60 \mathrm{rpm}$. After a $5 \mathrm{~s}$ countdown and without altering the mentioned parameters, subjects were asked to begin pedaling as fast as possible while receiving verbal encouragement throughout the test. Peak power, mean power, minimum power, time to peak power, relative peak power, relative mean power $(\mathrm{W})$ and fatigue index $\left(\mathrm{W} \mathrm{s}^{-1}\right)$ were calculated and recorded in an online data acquisition system. The rate of perceived exertion (RPE) was collected after each trial using a modified 10-point Rating of Perceived Exertion Scale (CR-10:RPE) (Borg, Hassmén, \& Lagerström, 1987). Capillary blood samples were taken on right fingertips at the third minute after completion of the test and analyzed for lactate concentration by using the Lactate Pro (LT-1710, Arkray, Japan) portable analyzer.

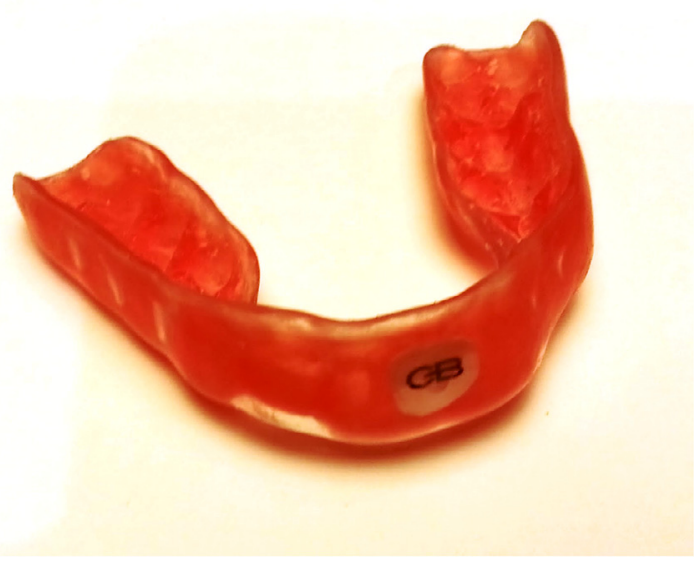

Fig. 1. Type of mouthguard used for the study. 


\subsubsection{Airflow dynamics measurements}

While in standing position, subjects were asked to breath at two different paces (NON-FORCED and FORCED) during 30-s under three different conditions: open mouth without mouthguard, jaw clenching without mouthguard and jaw clenched with mouthguard (OMNM, JCNM and JCM, respectively). In NON-FORCED breathings, the subjects were asked to breath in a resting pace (approximately at 30 inspirations per minute). In FORCED condition, subjects were asked to breath as quick and deep as possible. Rest time was 3 min after both measurements under each pace and 6 min between conditions. Conditions were randomly distributed. A AFT21 non-rebreathing T valve and a AFT9 mouthpiece with the AFT4 bacterial filter were mounted on a AFT25 facemask and was connected to a Biopac MP100 system through the wide-range airflow transducer amplifier TSD107B (Biopac Systems, Inc., CA, United States). The system recorded variations in airflow during the exercise, and the software Acknowledge 3.0.9. (Biopac Systems, Inc., CA, United States) plotted and recorded the flows on a time scale. The sample rate was established at $200 \mathrm{~Hz}$. Peak air flow was detected and mean air flow during the $30 \mathrm{~s}$ interval was calculated for all conditions.

\subsection{Statistical analysis}

Statistical analyses were performed with the Statistical Package for Social Science version 22.0 (SPSS, Inc., Chicago, IL). The Shapiro-Wilks test was used to test for normal distribution of the data. To compare Wingate parameters according two conditions, a paired $t$-test was used. When the data did not follow a normal distribution, an equivalent nonparametric test was applied. Comparisons between the three different conditions for airflow dynamics were carried out using a mixed model for repeated measures ANOVA. A significance level of $p<.05$ was used for all tests.

\section{Results}

The mean contrast for dependent WAnT variables was tested through t-test for related samples. Results showed a significantly higher performance $(P<0.05)$ of MOUTHG in all variables (Table 1$)$.

The RPE data was not normally distributed and a non-parametric Wilcox test was used to test the mean differences. Results showed no significant differences $(P<0.05, W=0.84)$ between NO-MOUTHG $(7.89 \pm 0.83)$ and MOUTHG (7.75 \pm 0.75$)$.

Mean contrasts for airflow dynamic variables was tested using a mixed model of two-way repeated measures ANOVA. A within-subject analysis compared the performance of the three conditions (OMNM, JCNM and JCM) $\times 1$ type of breathing between-factor (FORCED and NON-FORCED). When a significant $F$ score was found, Bonferroni post hoc tests were used to test the pairwise differences between the performances under the three conditions.

Multivariate analysis of mean airflow showed a significant main effect for condition $\left(F_{(2.53)}=47.85, p<0.05 ; \eta_{\mathrm{p}}^{2}=0.64\right)$ and type of breathing $\left(F_{(2,53)}=38.75, p<0.05 ; \eta_{\mathrm{p}}^{2}=0.59\right)$. Univariate contrast show a significant main effect for condition on type of breathing $\left(F_{(1.89,97.21)}=49.77, p<0.05 ; \eta_{\mathrm{p}}^{2}=0.48\right)$. In this case, the sphericity assumption was violated and the number of degrees of freedom was adjusted using the Huynh-Feldt method. Also, the analysis of peak airflow show a significant main effect for condition $\left(\mathrm{F}_{(2,53)}=54.64, p<0.05 ; \eta_{\mathrm{p}}^{2}=0.67\right)$ and type of breathing $\left(\mathrm{F}_{(2,53)}=39.80, p<0.05 ; \eta_{\mathrm{p}}^{2}=0.60\right)$. Univariate contrast show a significant main effect for condition on type of breathing $\left(\mathrm{F}_{(2,108)}=34.67, p<0.05 ; \eta_{\mathrm{p}}^{2}=0.39\right)$.

Pairwise comparisons indicated significantly better performance in mean airflow for FORCED with respect to NONFORCED for the three conditions $(P<0.05)$. No significant differences were found between the three conditions in NONFORCED $(P>0.05)$. However, in FORCED, a significantly higher air volume was expired in subjects using OMNM as compared to JCNM and JCM, and significantly higher air volume was expired in JCM under the JCNM condition $(P<0.05)$ (Fig. 2$)$.

There were significant differences in FORCED peak airflow for OMNM as compared to JCM and JCNM $(P<0.05)$. In contrast to mean airflow, there were no significant differences between JCM and JCNM $(P>0.05)$.

Power analyses were performed for all the variables after participant recruitment using GPOWER software (Bonn FRG, Bonn University, Department of Psychology). The post hoc analysis (given $\alpha$, sample size, and effect size) demonstrated a power of $99 \%$.

Table 1

Comparisions of Wingate variables wearing mouthguard and no mouthguard.

\begin{tabular}{|c|c|c|c|c|}
\hline Wingate variables & $\begin{array}{l}\text { No Mouthguard } \\
\text { Mean (SEM) }\end{array}$ & $\begin{array}{l}\text { With Mouthguard } \\
\text { Mean (SEM) }\end{array}$ & $t_{(27)}$ & $r$ \\
\hline Mean power BW $\left(\mathrm{W} \mathrm{kg}^{-1}\right)$ & $9.01(.15)^{*}$ & $9.14(.15)$ & -2.55 & .44 \\
\hline Peak power BW $\left(\mathrm{W} \mathrm{kg}^{-1}\right)$ & $16.04(.47)^{*}$ & $16.69(.43)$ & -2.91 & .48 \\
\hline Time to peak (s) & $4.55(.08)^{*}$ & $4.2(.05)$ & 3.07 & .51 \\
\hline Fatigue $\left(\mathrm{W} \mathrm{s}^{-1}\right)$ & $31.10(1.53)^{*}$ & $28.32(1.41)$ & 2.93 & .49 \\
\hline Lactate $\left(\mathrm{mmol} \mathrm{L}^{-1}\right)$ & $11.91(.34)^{*}$ & $11.01(.35)$ & 2.35 & .45 \\
\hline
\end{tabular}

Abbreviations: $\mathrm{BW}=$ body weight; $\mathrm{SEM}=$ standard error of measurement; $t_{()}=t$-test value(degree of freedom); $r=$ statistical power.

* Indicates a significant differences between the two conditions. 


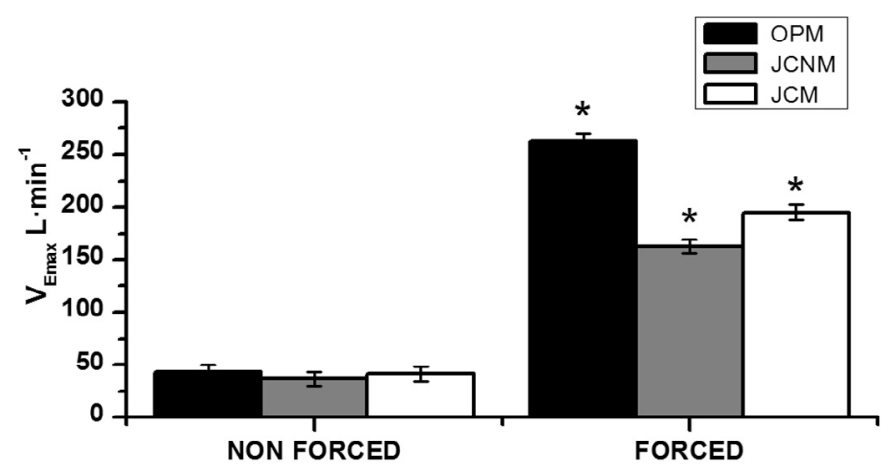

Mean Airflow $30 \mathrm{sec}$.

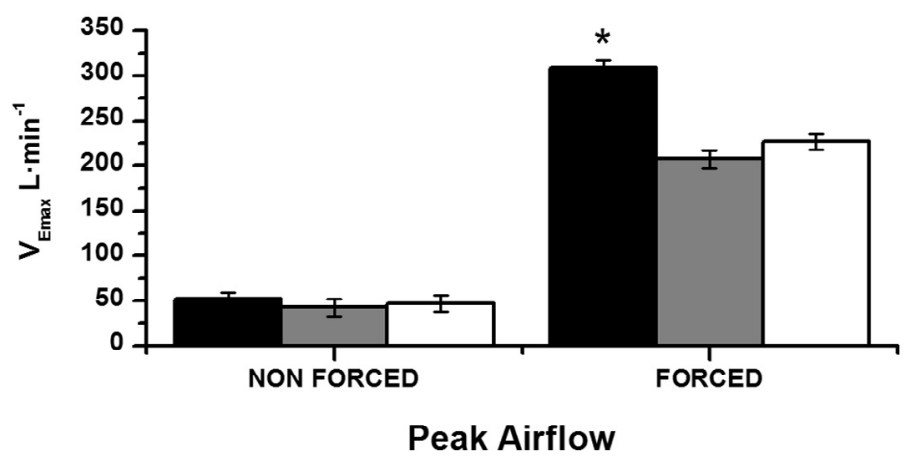

Fig. 2. Comparisions airflow measuraments between the three conditions. ONM = Open mouth without mouthguard, JCNM $=$ Jaw clenching without mouthguard, JCM = Jaw clenching with mouthguard. "Indicates a significant differences between the three conditions $(P<0.05)$.

\section{Discussion}

This study found significant differences in all variables of performance in WAnT. Subjects reached higher performances in MOUTHG condition for mean power body weight (BW $\left[\mathrm{W} \mathrm{kg}^{-1}\right]$ ), peak power BW $\left(\mathrm{W} \mathrm{kg}^{-1}\right)$, time to peak (s) and fatigue $\left(\mathrm{W} \mathrm{s}^{-1}\right)$. Also, subjects experienced significantly lower lactate accumulation $\left(\mathrm{mMol} \mathrm{L}^{-1}\right)$ under MOUTHG as compared to NO-MOUTHG conditions, but similar RPE. These findings may hold practical relevance for athletes whose sports require anaerobic effort, and especially for those athletes who need mouth protection.

The anaerobic ability test results of this study align with Arent, McKenna, and Golem (2010) which reported significant differences in WAnT peak and average power for athletes wearing a neuromuscular, dentistry-design mouthguard respect standard custom-fitted mouthguard condition. Arent et al. did not perform a comparison between an open-mouth condition and jaw clenching condition but indicated acute ergogenic effects caused by the neuromuscular mouthguard. This study did not use TENS to ensure relaxation of the lower jaw before the mouthguard fitting process; however some relaxation techniques were used. These previous mechanisms to improve the mouthguard fitting process improved the adjustability of the mouthpiece and may have served as a possible performance enhancer factor. Peak power and average power of the WAnT were also reported in similar studies (Cetin, Keçeci, Erdòan, \& Baydar, 2009; Jung, Chae, \& Lee, 2013). The effects of a RVC on the promotion of CAP is a possible explanation of the enhanced performance on anaerobic ability (i.e., both lactic and alactic). Peak and time to peak are consistent indicators of alactic anaerobic power and correlated with other power indicators such as CMVJ. Some studies reported ergogenic benefits of wearing mouthguards on jump performance (Ebben et al., 2008, 2010). Average power and fatigue index are used to explain the lactic anaerobic power of the subjects. Other studies have also reported ergogenic effects of wearing a mouthguard as compared to no mouthguard on different tests of lactic anaerobic performance in different populations (Cetin et al., 2009; Duddy et al., 2012; Dunn-Lewis et al., 2012). Milani et al. (2000) support the idea that jaw repositioning improves functional proprioception and promote benefits in movement and performance. According to Ebben et al. (2008) the combination of jaw clenching and RVC of lower body muscle groups increase the rate of force development and decrease blood lactate production. These conditions could enhance pedaling efficiency increasing the reflex activity of the Soleus, without energy expenditure, and reducing lactate production (Ebben et al., 2008). Furthermore, the improvement on airflow during jaw clenching can increase aerobic metabolism in reduction of anaerobic lactate production (Garner \& McDivitt, 2009). 
While other studies have also reported differences between traditional boil-and-bite and custom-fitted mouthguards (Dunn-Lewis et al., 2012). In the present study, one limitation is that only the differences between mouthguard and nomouthguard conditions were established. No comparison between different types of mouthguard was done. However, in this study should be noted that the mouthguard fitting process provide a precise adjustment and comfortable design and resulted in good conditions for powerful jaw clenching and taking benefits of the CAP. Indeed, the RVC of the jaw muscles seems to be facilitated by wearing a custom-fitted mouthguard and this consist a mechanism for CAP promotion. Therefore, the activation of muscles involved in powerful movements may be enhanced by CAP.

Exposing subjects to a $30 \mathrm{~s}$ maximal effort on a braked cycle ergometer produces a considerable amount of lactate that then accumulates in blood. Although subjects demonstrated significantly higher average power and lower fatigue, significantly lower blood lactate accumulations were found for subjects wearing a mouthguard. This finding is consistent with Bailey et al. (2014) and indicates that mouthguard use might create an advantage for sports where fatigue and lactate accumulations affects athletic performance. In accordance with the aforementioned study, we also did not found statistically differences in RPE.

This study found significant differences in airflow dynamics measurements (i.e., 30 s forced breathings under the same condition). To elucidate the potential mechanism involved with mouthguard use during intense exercise, patterns of $30 \mathrm{~s}$ mean airflow and peak airflow were assessed. This study's results differ from those of other studies that found no significant differences between use and no use of the two types of mouthguard on ventilatory parameters. This study found that both mean and peak airflow had a significant increase in JCM condition, as compared to JCNM and OMNM. Although prior studies used incremental tests, this study found similar mean airflow values at maximal intensities when comparing the values of FORCED condition with JCM and JCNM (Gebauer et al., 2011; Rapisura et al., 2010). These studies also observed no significant differences in ventilatory parameters between wearing and not wearing mouthguards under low intensity conditions; this study showed similar results.

This study showed how the wearability and comfort of the mouthguards improved forced expiratory airflow; these mouthguards could help to prolong exercise duration by improving ventilation and effort efficiency, as has been found in other studies (Garner, Dudgeon, \& Mcdivitt, 2011; Garner, Scheett, \& Mcdivitt, 2011; Rexhepi \& Brestovci, 2013). Moreover, Duarte-Pereira et al. (2008) showed that custom-fitted mouthguards have less interference with airflow than a boil-and-bite self-adapted mouthguards. According to the findings in this study, this contributes to the idea that custom-fitted mouthguards interfere less in ventilation. The respiratory patterns observed in this study showed that there are significant differences between jaw-clenching forced breathing wearing mouthguard respect non wearing it, assuming that open mouth situation offered better airflow dynamics (Garner, Scheett, \& McDivitt, 2011). A possible explanation for the improved ventilation during jaw clenching with mouthguard may be that custom mouthpieces improve jaw position by increasing oropharynx width and diameter (Garner, Dudgeon, \& McDivitt, 2011).

Further investigation is needed to compare use of different type of mouthpieces. Moreover, other athletic performance parameters should be tested in different sports and in women. Also, data of jaw clenching force might clarify the influence of different RVC magnitudes on the forces exerted by different prime movers in sports (Ebben et al., 2010). These findings could help practitioners improve an athlete's performance, particularly in sports where there are high-intensity anaerobic efforts that where high intensity action provokes jaw-clenching, thereby reducing lactate production and fatigue.

In conclusion, this study demonstrated that the use of custom-made, bite-aligning mouthguard had an ergogenic effect on WAnT variables, lactate production, and airflow dynamics in healthy young men compared with those who did not wear a mouthguard. This has important implications for athletes engaged in anaerobic efforts that cause jaw-clenching, and for those who need to reduce the incidence of dentoalveolar trauma.

\section{Acknowledgments}

The research team thank all of the study participants for their contributions. This research study was conducted with funds from the agreement between the Universitat Ramon Llull and the Departament d'Economia i Coneixement de la Generalitat de Catalunya. The authors declare that there is no conflict of interest between the researchers of this study and the participants, the materials and equipment used, or any other procedures undertaken during the experiments.

\section{References}

American Society for Testing and Materials (2000). Standard practice for care and use of athletic mouth protectors. West Consho-hocken (PA): Wiley.

Amis, T., Di Somma, E., Bacha, F., \& Wheatley, J. (2000). Influence of intra-oral maxillary sports mouthguards on the airflow dynamics of oral breathing. Medicine and Science in Sports and Exercise, 32(2), 284-290.

Arent, S. M., McKenna, J., \& Golem, D. L. (2010). Effects of a neuromuscular dentistry-designed mouthguard on muscular endurance and anaerobic power. Comparative Exercise Physiology, 7(02), 73-79.

Bailey, S. P., Willauer, T., Balilionis, G., Wilson, L., Salley, J., Bailey, E., et al (2014). Effects of an over the counter vented mouthguard on cardiorespiratory responses to exercise and physical agility. Journal of Strength and Conditioning Research, 29(3), 678-684.

Borg, G., Hassmén, P., \& Lagerström, M. (1987). Perceived exertion related to heart rate and blood lactate during arm and leg exercise. European Journal of Applied Physiology and Occupational Physiology, 56(6), 679-685.

Bourdin, M., Brunet-Patru, I., Hager, P.-E., Allard, Y., Hager, J.-P., Lacour, J.-R., et al (2006). Influence of maxillary mouthguards on physiological parameters. Medicine and Science in Sports and Exercise, 38(8), 1500-1504.

Cetin, C., Keçeci, A. D., Erdòan, A., \& Baydar, M. L. (2009). Influence of custom-made mouth guards on strength, speed and anaerobic performance of taekwondo athletes. Dental Traumatology, 25(3), 272-276. 
Chalmers, D. J. (1998). Mouthguards. Protection for the mouth in rugby union. Sports Medicine, 25(5), 339-349.

Collares, K., Correa, M. B., Da Silva, I. C. M., Hallal, P. C., \& Demarco, F. F. (2013). Effect of wearing mouthguards on the physical performance of soccer and futsal players: a randomized cross-over study. Dental Traumatology, 30(1), 55-59.

Duarte-Pereira, D. M. V., Del Rey-Santamaria, M., Javierre-Garcés, C., Barbany-Cairó, J., Paredes-Garcia, J., Valmaseda-Castellón, E., et al (2008). Wearability and physiological effects of custom-fitted vs self-adapted mouthguards. Dental Traumatology, 24(4), 439-442.

Duddy, F., Weissman, J., Lee, R., Paranjpe, A., Johnson, J. D., \& Cohenca, N. (2012). Influence of different types of mouthguards on strength and performance of collegiate athletes: A controlled-randomized trial. Dental Traumatology, 28(12), 263-267.

Dunn-Lewis, C., Luk, H.-Y., Comstock, B. A., Szivak, T. K., Hooper, D. R., Kupchak, B. R., et al (2012). The effects of a customized over-the-counter mouth guard on neuromuscular force and power production in trained men and women. Journal of Strength and Conditioning Research, 26, $1085-1093$.

Ebben, W. P. (2006). A brief review of concurrent activation potentiation: Theoretical and practical constructs. Journal of Strength and Conditioning Research, 20(4), 985-991.

Ebben, W. P., Flanagan, E. P., \& Jensen, R. L. (2008). Jaw clenching results in concurrent activation potentiation during the countermovement jump. Journal of Strength and Conditioning Research, 22(6), 1850-1854.

Ebben, W. P., Kaufmann, C. E., Fauth, M. L., \& Petushek, E. J. (2010). Kinetic analysis of concurrent activation potentiation during back squats and jump squats. Journal of Strength and Conditioning Research, 24(6), 1515-1519.

Ebben, W. P., Leigh, D. H., \& Geiser, C. F. (2008). The effect of remote voluntary contractions on knee extensor torque. Medicine and Science in Sports and Exercise, 40(10), 1805-1809.

Forgione, A. G., Mehta, N. R., \& Westcott, W. L. (1991). Strength and bite, Part 1: An analytical review. Cranio: The Journal of Craniomandibular Practice, 9(4), 305-315.

Francis, K. T., \& Brasher, J. (1991). Physiological effects of wearing mouthguards. British Journal of Sports Medicine, 25(4), $227-231$.

Garner, D. P., Dudgeon, W. D., \& Mcdivitt, E. J. (2011). The effects of mouthpiece use on cortisol levels during an intense bout of resistance exercise. Journal of Strength and Conditioning Research, 25(10), 2866-2871.

Garner, D. P., Scheett, T. P., \& Mcdivitt, E. J. (2011). The effects of mouthpiece use on gas exchange parameters during steady-state exercise in college-aged men and women. The Journal of the American Dental Association, 142(9), 1041-1047.

Garner, D. P., \& McDivitt, E. (2009). Effects of mouthpiece use on airway openings and lactate levels in healthy college males. Compendium of Continuing Education in Dentistry, 30, 9-13 (Jamesburg, N.J.: 1995).

Geary, J. L., Clifford, T. J., \& Kinirons, M. J. (2009). Occlusal accommodation and mouthguards for prevention of orofacial trauma. Oral Health E Preventive Dentistry, 7(1), 55-59.

Gebauer, D. P., Williamson, R. A., Wallman, K. E., \& Dawson, B. T. (2011). The effect of mouthguard design on respiratory function in athletes. Clinical Journal of Sport Medicine: Official Journal of the Canadian Academy of Sport Medicine, 21(2), 95-100.

Jung, J.-K., Chae, W.-S., \& Lee, K.-B. (2013). Analysis of the characteristics of mouthguards that affect isokinetic muscular ability and anaerobic power. The Journal of Advanced Prosthodontics, 5(4), 388-395.

Milani, R. S., De Periere, D. D., Lapeyre, L., \& Pourreyron, L. (2000). Relationship between dental occlusion and posture. Cranio, 18(2), 127-133.

Patrick, D. G., van Noort, R., \& Found, M. S. (2005). Scale of protection and the various types of sports mouthguard. British Journal of Sports Medicine, 39(5), 278-281.

Rapisura, K. P., Coburn, J. W., Brown, L. E., \& Kersey, R. D. (2010). Physiological variables and mouthguard use in women during exercise. Journal of Strength and Conditioning Research, 24(5), 1263-1268.

Rexhepi, A. M., \& Brestovci, B. (2013). The functional efficiency of mouthguards in martial sports. Human Movement, 14(4), 353-359. 